\title{
Field-Modulated Selective Ion Storage in a Quadrupole Ion Trap
}

\author{
Greg Wells and Chuck Huston \\ Varian Chromatography Systems, Walnut Creek, California, USA
}

\begin{abstract}
A new method of selective ion storage in a quadrupole ion trap is described. Broadband waveforms were applied to the endcaps of an ion trap to eject unwanted ions by resonance excitation, which enhanced the storage of selected target ions. A unique trapping field amplitude modulation technique allowed the use of waveforms with fewer frequency components. The requirements and methods of calculations for frequency-optimized waveforms are discussed. Advantages of this method include the reduction of target ion loss that results from collision-activated dissociation. In other applications, equivalent performance, relative to methods that use nonmodulated trapping fields combined with waveforms that have a higher frequency density, was shown. (J Am Soc Mass Spectrom 1995, 6, 928-935)
\end{abstract}

$\mathrm{T}$ he ion trap is a sensitive and versatile mass spectrometer. The storage of large numbers of ions will result in coulombic interactions that degrade the sensitivity and resolution of the device. The need to utilize the limited ion storage capacity of the trap has resulted in the development of several techniques to eliminate unwanted ions from the trap and selectively accumulate only specified target ions. $\mathrm{rf}-\mathrm{dc}$ isolation techniques have been used to selectively trap and accumulate ions [1-3]. These techniques establish trap conditions that destabilize unwanted ions. A significant limitation to this approach is that only a single contiguous range of target ions can be selectively stored.

Ions trapped in a quadrupolar trapping field have amplitude oscillations whose characteristic (or secular) frequencies are functions of the mass-to-charge ratio of the ion and the frequency and voltage of the rf trapping field [4]. The radial or axial motion of the ions can be increased, via resonance excitation, by subjecting the ions to a field whose frequency matches the characteristic frequency of the ion motion. The selectivity of resonance ejection was utilized in early studies of ion trapping processes to eliminate interfering ions [5]. In these studies a supplemental voltage was added to the endcap electrodes of the trap to create a dipole field whose frequency matched the secular frequency of the ion to be ejected. The ions in resonance with this field increased their amplitudes in the axial direction until the ions were ejected. Swept frequency techniques [6, 7] for ion ejection have met with limited success due to the low duty cycle for ejection. More recently, broadband resonance excitation techniques have been described that use stored waveform inverse Fourier

Address reprint requests to Greg Wells. Varian Chromatography Systems, 2700 Mitchell Drive, Walnut Creek, CA 94598. transforms (SWIFT) [8-12], constructed waveforms [13, $14]$, or filtered noise $[15,16]$.

The techniques that utilize broadband resonance excitation are all similar in that the waveforms have numerous frequency components to eject unwanted ions and notches in the frequency spectrum that correspond to the secular frequencies of the target ions. The waveforms are applied during the ionization period to continuously eject the unwanted ions. Efficient isolation of the target ions requires very narrow notches with frequency components on each side of the target ion secular frequency to eject the corresponding ions with masses above and below it. The coulombic influence of space-charge on the ion secular frequency, however, can shift the frequency as though a dc field were present [1]. Because there is a finite time required to eject unwanted ions from the trap by resonance excitation, there is some average space-charge, throughout the ionization period, in excess of that due only to the target ions. The space-charge can shift the secular frequency of the target ions from the theoretical value used to determine the center frequency of the notch. Thus, when narrow notches are used for isolation and a large amount of space-charge is present, the secular frequencies of many of the ions may be shifted outside of the notch, which causes these ions to be ejected.

More recently it was recognized that in nonideal ion traps that possess an octopole distortion, the secular frequency of the ion depends on the amplitude [17]. Thus ions formed far from the center of the trap (e.g., during electron ionization or ion injection processes) will have secular frequencies that are different from the values that they would have at the center of the trap. The use of a buffer gas in ion traps for collisional dampening of the ion oscillations is advantageous for both electron ionization $[4,5,18]$ and ion injection 
processes [19-22]. It would be expected that ions formed or injected tar off center followed by collisional dampening to the center would experience a time dependent shift in their secular trequencies. Again, the initial frequencies would be different than those located at the center of the notch, which causes ejection of some of the target ions.

This work used broadband waveforms combined with amplitude modulation of the fundamental rf trapping field to selectively store specified target ions and eject unwanted matrix ions. The field modulation technique was shown to produce results similar to previous methods while oftering several advantages. Amplitude modulation of the rf trapping field modulates the secular frequencies of stored ions. The dipolar field formed when a broadband frequency tailored waveform was applied to the endcap clectrodes of an ion trap was periodically brought into resonance with the secular frequencies of the ions, when the rf trapping voltage was modulated, and axial excitation resulted in ion ejection. The proper selection of the frequency components resulted in the ejection of unwanted ions and the selective accumulation of target ions.

\section{Experimental}

All experiments were performed via a Varian QISMS ion trap (quadrupole ion storage mass spectrometer; Varian Chromatography Systems, Walnut Creek, CA) and prototype selected ion storage (SIS) software. The QISMS system contained a built-in arbitrary waveform generator (Wave - Board) that applied a waveform to the end cap electrodes to create a dipolar field. Memory on the Wave Board allowed the storage of 32 different waveforms at any time. Additional scan functions and waveforms could be down-loaded to the instrument at specified times during data acquisition or in real time in instrument control. The QISMS software allowed the creation of user-defined scan functions with control of the rf and de voltage, as well as the application of broadband waveforms to any segment of the scan function. The modulation of the $\mathrm{rf}$ voltage was accomplished by software control of the rf digitalto-analog converter (DAC) by using a triangle modulation function. The sample used for most of the work reported here was perfluoro-tri-n-butylamine (FC-43). The pesticide samples of 100 -ppm aldrin and hepatachlor epoxide in hexane were obtained from PolyScience Corp. (Niles, IL). The trap was operated at a temperature of $150^{\circ} \mathrm{C}$. The approximate trap pressure was calculated to be 2.0 mtorr based on the trap conductance, pumping speed for helium, and the measured column flow rate of $1.0 \mathrm{~atm} \mathrm{~cm}^{3} / \mathrm{min}$.

\section{Results and Discussion}

\section{Trapping Field Modulation}

Figure 1 shows the field-modulated selective ion storage (FMSIS) scan function used in these studies. The

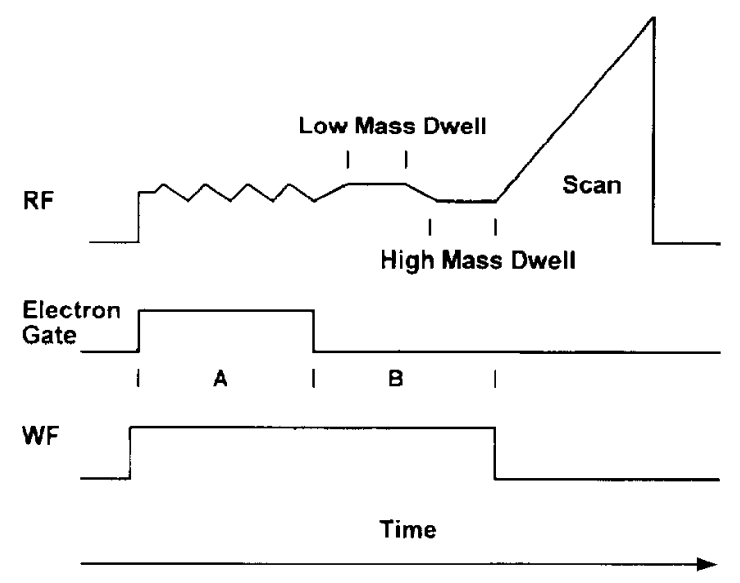

Figure 1. MSIS san function.

ionization period is defined by the electron gate. The broadband waveform was applied during the ionization period and for a specified time after the end of ionization. During the ionization period the amplitude of the rf storage field was modulated by $2 \%$ by using a triangle modulation function at a frequency of 1000 $\mathrm{H}$. A $2 \%$ modulation requires a $\pm 3 \mathrm{DAC}$ change in the rf trapping field when it is at a nominal value of 300 DACs; this corresponds to a low mass-to-charge ratio cutoff mass of 48 (i.e., the mass at which $q=$ 0.908 ). A modulation frequency of $1000 \mathrm{~Hz}$ requires a dwell time of $83 \mathrm{~ms}$ per DAC step. The collisional damping of an ion to the center of the trap takes several milliseconds $[4,5,18-22]$. Because the secular trequencies of ions in the trap are calculated from their theoretical values when they are located at the center (where the octopole effects on the secular frequencies are zerol, an additional "cool time" after the end of ionization is necessary to allow ions far from the center to be collisionally damped to the center. Therefore, after the end of ionization, the rf field completed one additional modulation cycle. At the extremes of each half cycle there were user-defined dwell times (low mass dwell, high mass dwell) that allowed fine tuning of the resonant ejection process on each side of the target ions. Without the cool time, unwanted ions formed at the end of ionization would not be completely ejected. Normal scanning of the ions from the trap for detection followed the last modulation cycle. This scan funclion was used for both the automatic gain control (AGC) pre-scan [23] and the analytical scan.

The motion of an ion in a quadrupole field can be determined from the solution to the Mathieu equation [4]. The stable solutions to the equation are characteri $<$ ed by the parameters $q$ : and $a$; that define the operating point of the ion within the stability region. These parameters are defined as [4]

$$
\begin{aligned}
& q_{z}=-8 c V / m\left(r_{0}^{2}+2 z_{10}^{2}\right) / \Omega^{2} \\
& q_{z}=-16 c U / m\left(r_{i}^{2}+2 z_{0}^{2}\right) / \Omega^{2}
\end{aligned}
$$


where $V$ is the amplitude (1) to peak) of the rf potential applied to the ring electrode, $U$ is the do potential, $m$ is the mass-to-charge ratio $\left(m /=1, r_{n}\right.$ is the radius of the ring electrode, $z_{11}$ is the inscribed radius of the endcap electrodes, and $\Omega$ is the rf drive frequency. The secular frequency of an ion $(1)$ can be determined from the value of $\beta=[4]$ :

$$
\omega_{-}=(\beta / 2) \Omega
$$

The value of $\beta$, is a function of the operating point in $(a, q)$ space and can be computed from a well known continuing fraction [3]. In this work no do field was applied, thus $a_{-}=0$.

Changing the amplitude of the trapping field by $\lrcorner \mathrm{V}$ caused a change in the operating point $J i$ and, thus, the secular frequency of the ion $\lrcorner \omega$. Because the secular frequency changes with mass, the change in frequency caused by a change in trapping field also was a function of mass. A calculation of the frequency change as a function of mass for a $2 \%$ rf voltage change and a low mass-to-charge ratio cutoff mass of 48 , indicated that $m /=80$ shifted by $5 \mathrm{kHz}$ and $m /=$ 650 shifted by $0.2 \mathrm{kl} \mathrm{z}$. The frequency shift is highly nonlinear, it changes rapidly at low mass and only slightly changes at high mass. This is the result of the inverse mass dependence of the operating point $\eta$, and the nonlinear relationship between of and the secular frequency. When the frequency components in the waveform were properly spaced the secular frequencies of the unwanted ions were periodically resonantly excited and ejected as the trapping field amplitude was varied.

\section{Wareform Calculations}

Figure 2 a illustrates the separation between secular frequencies that corresponds to an increasing range of integer masses. Figure $2 b$ shows the frequency components of a waveform that would be constructed for use

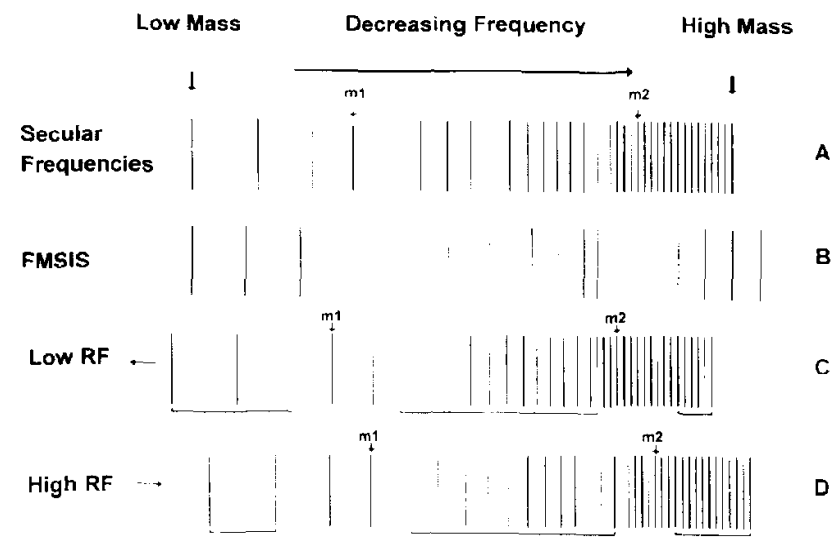

Figure 2. (a) Secular frequency spacing for integer masses, (b) Frequency spacing for the FMSIS waveform. (c) Shift in secular frequencies that result from lowering the amplitude of the rt storage voltage. (d) Shift in secular frequencies that results from increasing the amplitude of the rf turage voltage. with a modulated trapping field to selectively trap masses $m 1$ and $m 2$. Without field modulation, several ions would not have secular frequencies in resonance with the frequency components in the applied waveform. Figure $2 \mathrm{c}$ and $\mathrm{d}$ show how modulation (lowering and raising) of the trapping field shifts the secular frequencies of the ions. During each complete cycle of modulation, the secular frequencies of the unwanted ions are swept through one of the frequency components of the applied waveform. Comparison of Figure $2 c$ and $\mathrm{d}$ with $2 \mathrm{~b}$ also shows that $m 1$ and $m 2$ never leave the frequency notch, whereas all the unwanted ions do and thus are resonantly excited and ejected. The advantage of this approach is that it allows the effective notch width to be changed in real time by changing the amplitude of $\mathrm{rf}$ modulation, without the need to change the waveform.

The method of calculating a field modulated waveform for a single mass range, could easily be extended for use with multiple mass ranges. Given that the mass range of target ions to trap is bounded at each end by $m_{1}$ (low mass) and $m_{\mathrm{H}}$ (high mass), the corresponding secular frequencies, $f_{\mathrm{mL}}$ and $f_{\mathrm{mH}}$, at each end of the contiguous range of target ions could then be computed. If only a single mass was to be isolated, then $m_{\mathrm{I}}=m_{\mathrm{H}}$ and $f_{\mathrm{mL}}=f_{\mathrm{mH}}$; this was defined to be an isolation window of 1 . An isolation window of 5 would, therefore, be $m_{\mathrm{mH}}=m_{\mathrm{ml}}+4$. Resonance energy was absorbed from the supplemental dipole field over a finite frequency. If the width of the resonance was defined to be $\Delta f_{r}$, then an "edge scaling" factor could be defined as

$$
f_{\mathrm{es}}=\Delta f_{\mathrm{r}} / 2
$$

The edge scaling factor defined the minimum notch width that could be obtained (i.e., when $m_{\mathrm{L}}=m_{\mathrm{H}}$, and $f_{\mathrm{mL}}=f_{\mathrm{mH}}$ ) and was itself a function of mass. Thus, the frequencies at each end of the notch, for the nonmodulated field case, would be:

$$
\begin{aligned}
& f_{\mathrm{L}}=f_{\mathrm{mL}}+f_{\mathrm{es}}=f_{\mathrm{mL}}+\Delta f_{\mathrm{r}} / 2 \\
& f_{\mathrm{H}}=f_{\mathrm{mH}}-f_{\mathrm{es}}=f_{\mathrm{mH}}-\Delta f_{\mathrm{r}} / 2
\end{aligned}
$$

The change in the secular frequencies $\Delta f_{\mathrm{L}}$ and $\Delta f_{H}$ that resulted from a change in the trapping field can be computed for eqs 5 and 6 . These values yield the final frequencies that define the ends of the notch for the field modulated case:

$$
\begin{aligned}
& f_{\mathrm{el}}=f_{\mathrm{L}}+\Delta f_{\mathrm{l}}=f_{\mathrm{mL}}+\Delta f_{\mathrm{r}} / 2+\Delta f_{\mathrm{L}} \\
& f_{\mathrm{cH}}=f_{\mathrm{L}}-\Delta f_{\mathrm{H}}=f_{\mathrm{mH}}-\Delta f_{\mathrm{r}} / 2-\Delta f_{\mathrm{H}}
\end{aligned}
$$

Once the end frequencies were determined, the frequencies outside of the notch could be established. A complete set of frequencies could be found that would, in the absence of any notches, eject all ions over the entire mass range, from the low mass-to-charge ratio cutoff mass to a defined upper limit. This could be 
done by dividing the mass range into multiple regions. Within each region the smallest frequency change within that region determined the frequency spacing for the waveform components that span the range. For example, to eject ions from $m /=500-600$, frequencies spaced $1.5 \mathrm{kHz}$ apart were sufficient; to eject ions from $m /=50-75$, spacing of $5 \mathrm{kHz}$ could be used. A waveform that has as few as 1.30 components, spaced properly, was found to easily eject ions that spans the entire mass range from $m /=48-650$ when the nominal low mass-to-charge ratio cutoff mass was 48 and the trapping field was modulated by $2 \%$. A random selection of phases ensured that the dynamic range of the wareform was small-typically $15 \mathrm{~V}$ peak to peak for the unnotched waveform.

\section{Amplitude Tailoring of Frequency Components}

The required amplitude of each frequency component was determined by measuring the minimum voltage necessary to eject $95 \%$ of the ions for a given mass-tocharge ratio. The ions were first isolated by applying a waveform during ionization and for a 5 -ms cooling time after ionization ended. A supplemental axial voltage was then applied for an additional 5-ms followed by the normal mass scan. The scan was repeated and the frequency of this supplemental voltage was varied slowly so that during the 5 -ms excitation period it was essentially constant. This produced a frequency scan through the resonance and was repeated at progressively larger voltages. In this way the frequency adsorption profile could be determined as a function of voltage for mass-isolated ions. The width of the resonance at half-maximum for the isolated ion at the minimum ejection voltage was observed to range from approximately $1200 \mathrm{~Hz}$ for low masses (below $\mathrm{m} / \mathrm{s}$ 100) to $800 \mathrm{~Hz}$ for high masses (above $m / z 500$ ). Figure 3 shows the relationship between amplitude and mass at different $r f$ trapping voltages, expressed in terms of the low mass-to-charge ratio cutoff mass. The

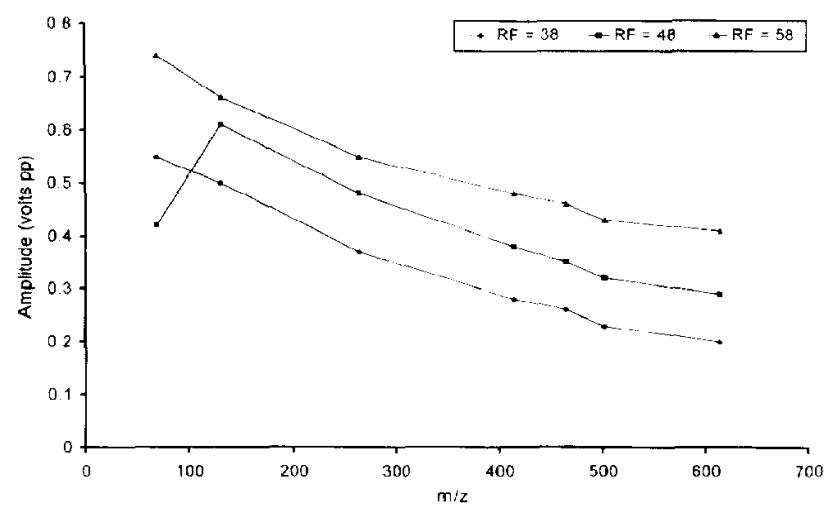

Figure 3. Minimum axial voltage (peak to peak) required to eject the mass-isolated targest ion as a function of mass and $r^{\circ}$ storage mass. datum point for $m / z 69$ and a cutoff mass of 48 corresponded to a value of $\beta=0.491$. This $\beta$ is very close to a nonlinear resonance of the trapping field [17, 24 , which has been shown to lower the voltage required to eject an ion located at that operating point. The data were collected by using the maximum fixed ionization time that still would give unit mass resolution for each test ion. Ionization times less than the maximum showed no change in the required voltage. Excitation times of 5,3 , and $1 \mathrm{~ms}$ were investigated. As the excitation time was reduced, the minimum voltage was increased linearly such that the fluence (the product of the amplitude and time) [25] was essentially constant. Therefore, the voltage scaling as a function of mass did not change with either ionization time or space-charge level.

\section{Effects of Low Mass-to-Charge Ratio Cutoff Mass and Space-Charge}

The effects of $\mathrm{rf}$ storage voltage and space-charge were studied by operating the trap in two different modes. In both modes the scan function consisted of three consecutive time periods $A$ and $B$, as shown in Figure 1, and an additional period $C$. Period $C$ was inserted after period $B$ and prior to mass scanning. In modes 1 and 2 , period $A$ represents the ionization time defined by the electron gate. The ionization rf storage voltage, expressed as the low mass-to-charge ratio cutoff mass, was $m / z 48$ for all measurements. In mode 1 a waveform was applied during the ionization period as well as period B. Period $B$ provided an additional 5 -ms cool time (during which the rf storage voltage was the same as that during the ionization period) to isolate the target ion and to eject all other ions. The rf storage voltage was varied during the subsequent period $\mathrm{C}(5 \mathrm{~ms}$ in duration) and followed by the normal mass scan to measure the ion intensities. In mode 2 no isolation occurred during ionization and the rf storage voltage was varied during period $B$, also without mass isolation. Mass isolation occurred in period $C$ during which the rf voltage was the same as that during ionization (low mass-to-charge ratio cutoff mass $=48$ ); the normal mass scan followed.

In mode 1 the effects of storage if on the trapping efficiency could be determined in the absence of space-charge due to other ions. In mode 2 the added effect of space-charge on the trapping efficiency could be measured. Measurements were made of normalized intensity (intensity divided by ionization time) at each rf level, for three different fixed ionization times, and for test ions $m / z 69$ and 464. At storage levels above $m / z 25$ with high mass ions there was little dependence between trapping efficiency and the low massto-charge ratio cutoff mass or space-charge (see Figure 4 , where "t $=5000$, iso" represents an ionization time of $5000 \mu \mathrm{s}$ for the mass-isolated test ion). The loss of storage cfficiency occurred at lower of levels and was 


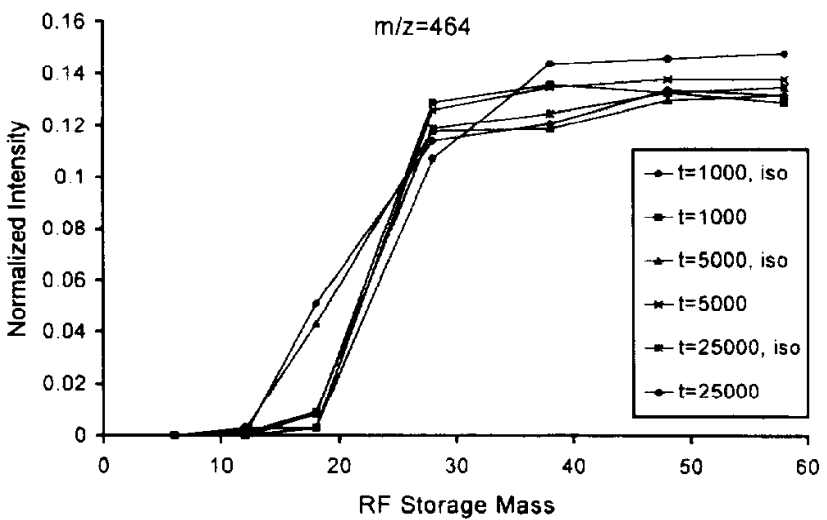

Figure 4. Normalized ion intensities as a function of $\mathrm{rf}$ storage mass for target ion $m / z 464$.

more pronounced with higher space-charge (larger ionization times). Similar data were obtained for $\mathrm{m} / \mathrm{z}$ 69, where no measurable affect of space-charge could be observed down to a cutoff mass of $m / z=10$. Below $m / z=10$ the storage efficiency quickly fell to zero. The complete absence of mass isolation before the mass scan allowed space-charge levels from longer ionization times to be large enough to result in a severe loss of resolution.

The individual effects of the broadband waveform and the trapping field modulation are shown in Figure 5. Figure 5a shows the electron ionization (EI) spectrum of FC-43 with an AGC ionization time of $806 \mu \mathrm{s}$. Figure $5 \mathrm{~b}$ shows the effect of application of the FMSIS waveform constructed to isolate $m / z 464$. The ioniza-

a

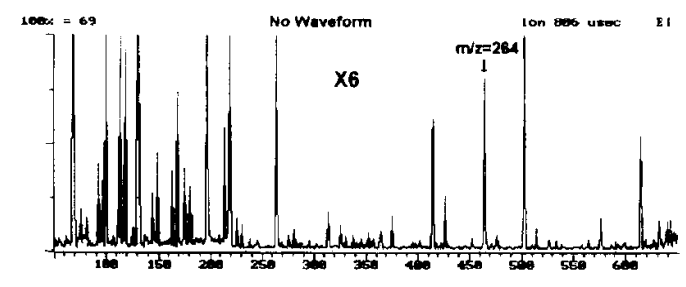

b

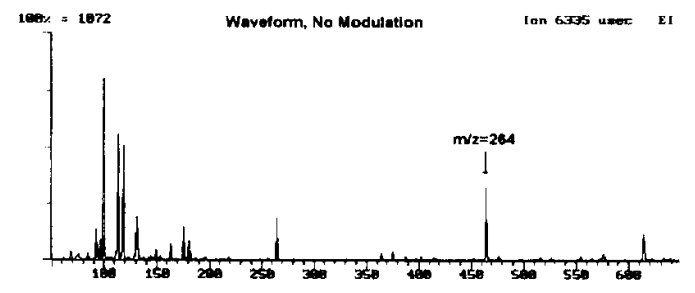

c

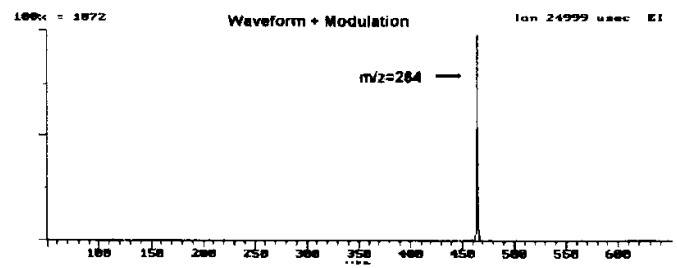

Figure 5. (a) FC-43 spectrum without waveform applied to endcaps. (b) Spectrum with waveform to isolate $m / z 464$ but no field modulation. (c) Spectrum with waveform and field modulation.

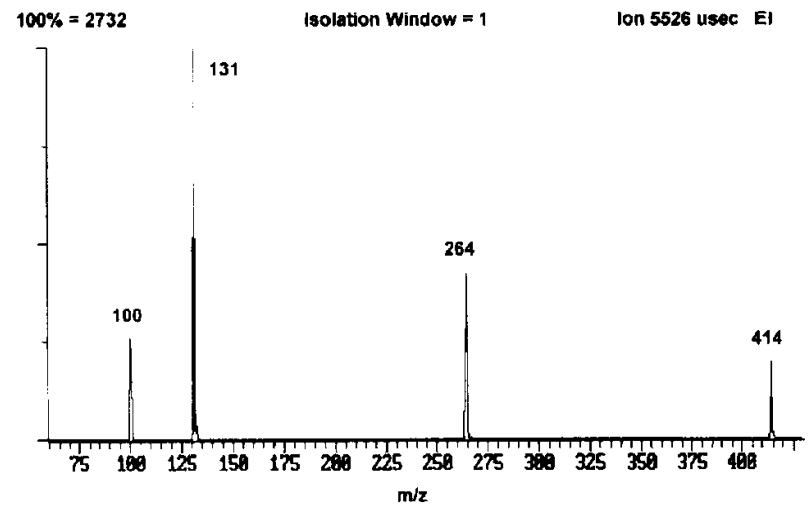

Figure 6. Selective storage of $m / z 100,131,264$, and 414 from FC -43 by using an isolation window of 1 .

tion time was increased to $6335 \mu \mathrm{s}$ and many, but not all, of the unwanted ions were ejected. Figure $5 c$ shows the result of the combined effects of the waveform and a $2 \%$ amplitude modulation of the trapping field. All of the ions except for $m / z 464$ were ejected and the AGC ionization time was increased to the maximum allowable value of $24,999 \mu \mathrm{s}$.

Figure 6 shows the isolation of four ions of FC -43 by using an isolation window of 1 (1-u wide isolation). Increasing the isolation window to 5 (5-u wide isolation) showed no change in the observed intensities or relative intensity ratios for the isolated ions. Figure 7 shows the region around $m / z 131$ without FMSIS. The inset figure shows the isolation of the $\mathrm{m} / z 132$ isotope from $m / z 131$. The scale was the same $(100 \%=528)$ and the AGC ionization time was increased to $25 \mathrm{~ms}$. It should be recognized that this type of mass isolation resolution (unit mass) cannot in general be obtained at higher mass. The secular frequencies for $m / z 131$ and 132 are 124.9 and $123.9 \mathrm{kHz}$, respectively, for a cutoff mass of $m / z$ 48. Therefore, a notch width of $1 \mathrm{kHz}$ could resolve masses separated by $1 \mathrm{kHz}$. At $m / z 600$ the same $1-\mathrm{kHz}$ notch would span a mass range of 22 .

In other methods of ion isolation utilizing notched broadband waveforms, the frequency components lo-

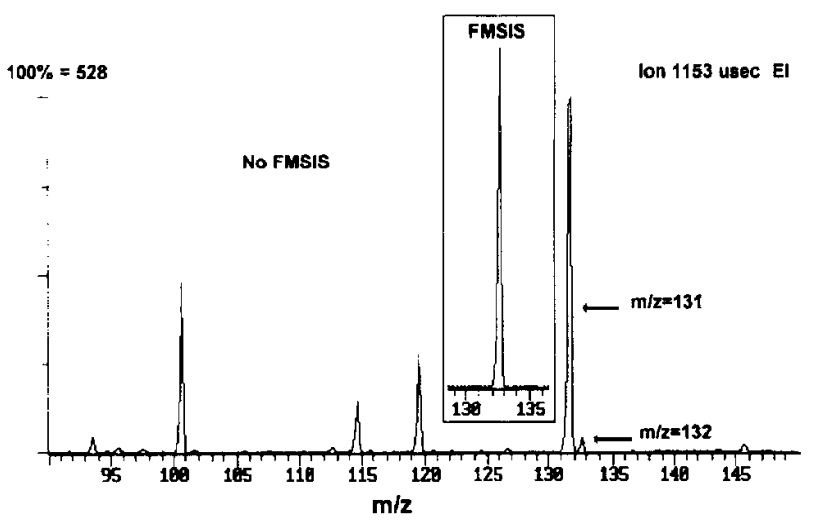

Figure 7. Spectrum of FC-43 in the region around $m / z 131$ without FMSIS. The inset shows the effect of applying the FMSIS waveform during ionization. 
cated on either side of the secular frequency of the target ion maintain a fixed separation from that frequency. In the field-modulated method the separation changes periodically so that the minimum separation occurs for only a short time. When the end frequencies of the notched waveform are in constant proximity to the secular frequency of the target ion, a small amount of energy is expected to be continuously coupled into the ion motion. This is similar to the conditions that are used in tandem mass spectrometry experiments in the ion trap to achieve collision-activated dissociation (CAD).

Because the loss of target ions from the notch by CAD would be expected to reduce sensitivity, the normalized intensity of $m / z 134$ from $n$-butyl benzene was monitored as a function of the waveform time. Butyl benzene easily dissociates in an ion trap to form predominantly $m / z 91$ and 92. A fixed ionization time of $5 \mathrm{~ms}$ was followed by a cool time of $5 \mathrm{~ms}$ to allow the ions to collisionally cool to the center of the trap. Waveforms then were applied to the trap for various times. Waveforms were constructed for a static rf field (not modulated) by co-adding frequency components from $20-450 \mathrm{kHz}$, spaced $500 \mathrm{H} / \mathrm{c}$ apart, with constant amplitudes, and with random phasing. The waveforms for the modulated case were constructed so that the "effective" notch width (notch width when the rf field was modulated) was the same as for the static case. Figure 8 compares the results obtained with FMSIS waveforms (labeled Modulated in Figure 8) with those obtained without modulation (labeled Static) for notch widths of 3.5 and $7.5 \mathrm{kHz}$. The relative intensity (intensity with waveform divided by the intensity without waveform) for $m / z 134$ decreased slowly with increasing waveform time for a notch width of $7.5 \mathrm{kHz}$. The relative intensity for $m / z 134$ was observed to decrease dramatically for the static method when the notch width was reduced to $3.5 \mathrm{kHz}$, whereas the decrease in relative intensity for the modulated (FMSIS) method was much smaller. Both methods converged to similar values at shorter ionization times.

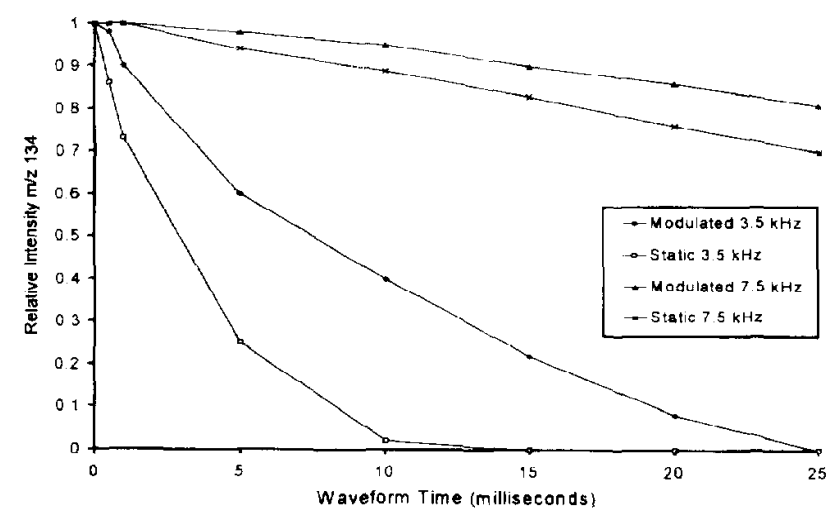

Figure 8. Relative intensities of $m /=134$ from $n$-butyl benzent versus waveform time for modulated waveforms and static field waveforms at notch widths of 3.5 and $7.5 \mathrm{kHz}$. Intensities at each time are relative to the intensities obtained without waveforms.
The effects of modulation frequency and helium bath gas pressure were studied briefly by monitoring the relative intensity of $m / z 134$ from $n$-butyl benzene, as a function of ionization time at numerous modulation frequencies and trap pressures. The highest usable modulation frequency was limited by the $\mathrm{rf}$ control loop bandwidth to $1200 \mathrm{~Hz}$. Changing the modulation frequency changes the rate at which the resonance maximum is passed and thus the amount of energy that is coupled into the ion motion (assuming a fixed amplitude for the waveform). Frequencies between 400 and $1200 \mathrm{~Hz}$ showed almost no effect on the normalized data when the waveform amplitude was scaled in direct proportion to the frequency to maintain a constant fluence. Frequencies below $400 \mathrm{~Hz}$ were not studied. No effect on helium bath gas pressure was observed over a range of 1-3 mtorr other than the expected increased loss of intensity at higher pressure due to increased CAD.

The effect of dynamic adjustment of the notch width by changes in the modulation amplitude was investigated. A $2 \%$ modulation of the rf storage voltage was utilized during the ionization period, followed by a cool time and one additional cycle by using a $2.65 \%$ modulation amplitude. An ion of $m / z 133$ from polysiloxane column bleed was used as the test ion. Figure 9a compares FMSIS to a broadband waveform for a static rf field (BB) constructed as previously described with the notch centered at the secular frequency of $m / z$ 133. The effective notch width for FMSIS during the postionization cycle was equal to the notch width for $B B(6.5 \mathrm{kHz})$. The ion intensities for the test ion were observed to be nearly the same. The effect of a decrease in the notch width to $2.5 \mathrm{kHz}$ for each waveform can be seen in Figure 9b. FMSIS showed a $14 \%$ loss of sensitivity when the resolution was increased, whereas the $\mathrm{BB}$ method showed a decrease of $76 \%$. The differences between the FMSIS and BB became less significant as the width of the notches was increased.

The application of FMSIS to determine aldrin and heptachlor epoxide in extracts of bell pepper was investigated. A matrix of bell pepper, extracted with methylene chloride and spiked with the analytes at the 100 -ppb level, was analyzed. The sample preparation method is described in detail by Feigel [26, 27]. Two mass ranges were stored for each compound: $250-270$ and 285-305 for Aldrin and 305-325 and 345-365 for heptachlor epoxide. The Wave $\sim$ Board control software was time programmable so that up to 200 different waveforms could be applied throughout the chromatogram at time intervals as small as $1 \mathrm{~s}$. The FMSIS waveform used for each compound was time programmed so that the appropriate waveform was applied during the retention time window for each compound. Analysis of the extract with $50 \mathrm{pg}$ on column failed to produce a detectable peak (monitoring $\mathrm{m} / \mathrm{z}$ 293 for Aldrin and $m / z 353$ for heptachlor epoxide) in the full scan EI mode (without a FMSIS waveform). 


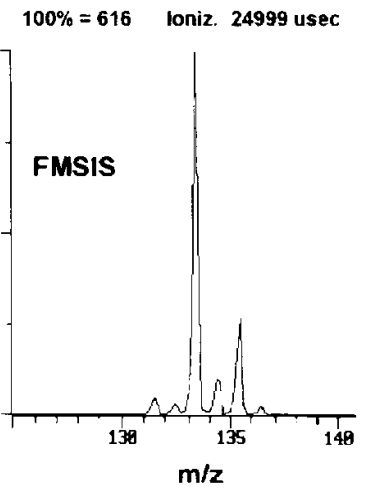

b

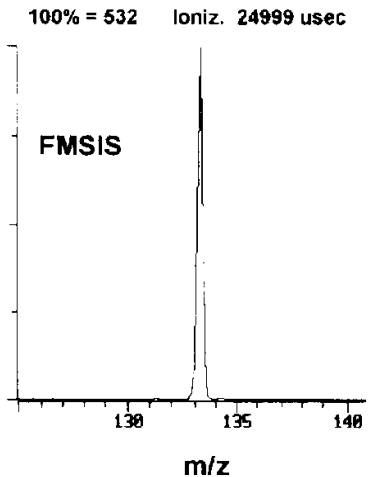

$100 \%=585$ loniz. 24999 usec
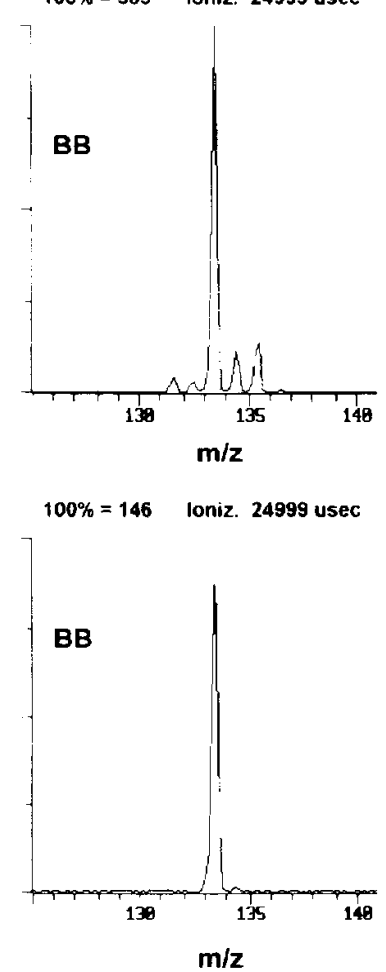

Figure 9. (a) Isolation of $\mathrm{m} /=1.33$ with a noteh width of $6.5 \mathrm{kl} / \mathrm{l}$ for FMSIS and BB. (b) Notch width of $2.5 \mathrm{kH} \%$.

Figure 10 shows the same EI spectrum that utilizes FMSIS to eliminate the background matrix ions and selectively store the designated mass ranges for each compound. The improvement in detection was the result of ejection of the ions outside of the specified storage ranges, which allowed a dramatic increase in the ionization time and, hence, the number of analyte target ions that were in the trap. For this analysis, relatively wide mass ranges nere stored so that the

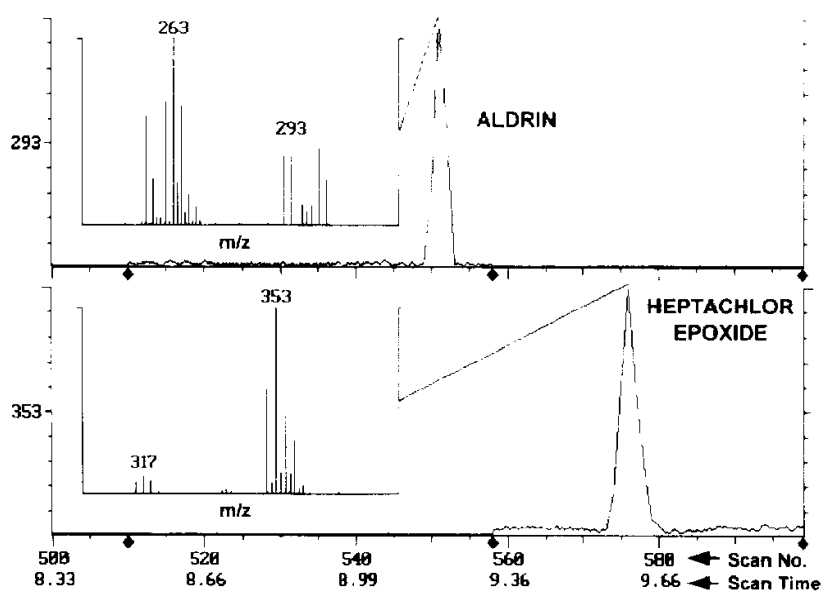

Figure 10. FMSIS mode reconstructed single ion chromatograms of aldrin $(m / 2=243)$ and heptachlor epoxide $(m /=$ 353 ) in bell pepper. Selective storage of mass ranges 250-270 and 285-305 for aldrin and $305-325$ and $345-365$ for heptachlor epoxide.

qualitative information of the isotopic clusters could be retained. The spectral characteristics were the same as the neutral concentration changed over several orders of magnitude. Feigel $[26,27]$ has reported library searchable detection limits for pesticide residues below $10 \mathrm{pg}$ on column (10:1 signal-to-noise ratio) with FMSIS, with over 4 orders of magnitude of linear response. The consistency of the spectra and the response linearity (even for the narrowest mass isolation range where space-charge effects are the greatest) is the result of holding the space-charge in the trap relatively constant by application of the waveforms during the $\mathrm{AGC}$ pre-scan. This allowed the analytical ionization time to adjust to changing neutral concentrations and to maintain a constant charge in the trap based on the jons in the isolated mass ranges.

\section{Conclusions}

The use of selective ion storage in conjunction with automatic gain control gave increased sensitivity for the target ions that resulted from increased ion production via increased ionization time. For low massto-charge ratio cutoff masses above 30 , there was no significant influence of ion storage capacity due to the presence of excess space-charge. The dominant effect of space-charge was to degrade the mass resolution of the target ions in the trap. It was not necessary, therefore, to simultaneously eject all unwanted ions from the trap in order to increase sensitivity. The trapping field-modulated approach allowed the construction of waveforms that contained a minimal number of components, which reduced the dynamic range of the waveform as well as the calculation time. The modulation technique has the additional advantage of allowing the effective notch width to be changed without changing the frequency composition of the waveform. In some applications the field modulation method was found to reduce the amount of target ion losses (during ion formation and isolation) due to CAD. The advantages of the field modulated selective ion storage technique enabled useful analysis of complex samples.

\section{Acknowledgments}

The authors wish to thank the reviewers for their constructive comments during the preparation of this manuscript.

\section{References}

1. Fulford, J. E.; March, R. F. Int. I. Mass Spectrom. Ion Phys. $1978,26,155$.

2. Eades, D. E.; Yates, N. A., Yost, R. A. Proceedings of the 39th ASMS Conference on Mass Spectrometry and Allied Topics; May $19-24,1991 ; p 1491$

3. Weber-Grabau, M. US Patent 4,818,869, 1989.

4. March, R. E.; Hughes, R. J. Quadrupole Storage Mass Spectrometry; Wilev: New York, 1991. 
5. Fultord, J. F.; How, D. ..; Hughen, R. J.; March, R. E.; Bonner R. F.; Wong, (i. I. I. Vin Set. Tirhol 1980 17(t), 829-835.

6. McLuckey, S. A. Gocringer, D. E.; Glish, (i. L. I. Am. Sor. Mass Spectrom. 1991, 2, 1121.

7. Schwartz. I.C.; Jartine, I. Retud Commmn. Mass Spetrom, 1992. 6., 313 .

8. Marshall, A. Ci.; Ricia, I. I..; Mang, T. L. LS J'atent +7 h1,547, 1988.

9. Goodman, S.: Hanna, A. LS Patent 4,445.234, 1990.

10. Cuan, S.; Marshall, A. (i. Amh Chm 1993, 63, 1288-1294

11. Jullian, R. K. Cooks, R. K. Amal. Chom. 1993, 65, 1827-1833.

12. Soni, . . H.; Cooks, R. Li Anat. (h'm. 1994, th, 2488 2496.

13. Franzen, I.; Gabling, R. H. Furopean Patent Application EP () 362432 A1, 1988.

14. Shaffer, B. A.; Karnichy, I.; Buttril, S. E. Ir. Procedtinge of the

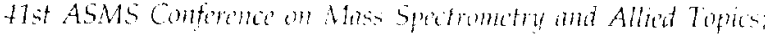
May 31-June t, 1493, pp S(12a 812b

15. Carrett, A. W.; Cisper, M. E., Nogar, P’. H.; Hemberger, P. H. Rapid Commun. Moss Spectron. 1994, is, 174.178

16. Kelley, P. F; Hoekman, D); Bradshaw, S. Procedinges of the Hat ASMS Conference on Miss Spectrometry and Alled Toples: May 31 June 4 , 1993, +5.3a $+53 b$.

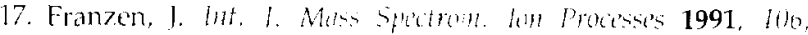
$6.3 \cdots 78$
18. Stafford, G. C.; Kelley, ['.E.; Stephens, D. R., US Patent $4,540,884,1985$.

19. Van Berkel, G. J.; Glish, G. L.; McLuckey, S. A. Anal. Chem. 1990, 62, 1284-1295.

20. Louris, J. N.; Amy, J. W.; Ridley, T. Y.; Cooks, R. G. Int. J. Muss Spectrom. Lon Processes 1989, 88, 97.

21. McLuckey, S. A.; Glish, G. A.; Asano, K. G. Anal Chim. Acta $1989,225,25$

22. Pedder, R. E.; Yost, R. A.; Weber-Grabau, M. Proceedings of the 37th ASMS Conference on Mass Spectrometry and Allied Topics; May 21-26, 1989, p 468

23. Stafford, G. C.; Taylor, D. M.; Bradshaw, S. C.; Syka, J. E. P.; Uhrich, M. D. Proceedings of the 35th ASMS Conference on Mass Spectrometry and Allied Topics; 1987, p 775.

24. Eades, D. M.; Johnson, J. V.; Yost, R. A. I. Am. Soc. Mass Spritrom. 1993, 4, 917-929.

25. March, R. E.; Londry, F. A.; Alfred, R. L.; Todd, J. F. J.; Penman, A. D.; Vedel, F.; Vedel, M. Int. I. Mass Spectrom. Ion Procisie's 1991, 110, 159-178.

26. Fiegel, C. Varian Application Note 21, Varian Chromatography Systems, Walnut Creek, CA, 1994.

27. Fiegtel, C. Varian Application Note 22, Varian Chromatographis Svistems, Walnut Creek, CA, 1994. 